\title{
Efeitos da meditação sobre os sintomas da ansiedade: uma revisão sistemática
}

\author{
Effects of meditation on anxiety \\ symptoms: a systematic review
}

\section{Júlia Lago Peixoto' 1 (1) Bruna Guimarães ${ }^{2}$ (1) Mara Dias Pires ${ }^{3}$ (1) Ítalo Antônio de Jesus Almeida Júnior ${ }^{4}$ (1)}

\author{
'Autora para correspondência. UNIFACS (Salvador). Bahia, Brasil. julialagopeixoto@gmail.com, \\ 2,4UNIFACS (Salvador). Bahia, Brasil. brunabritoguimaraes@gmail.com, yalmeidajunior@outlook.com \\ 3UNIFACS (Salvador), Escola Bahiana de Medicina e Saúde Pública (Salvador). Bahia, Brasil. mara.pires@unifacs.br
}

\begin{abstract}
RESUMO | INTRODUÇÃO: A Ansiedade é um estado subjetivo de medo, apreensão ou tensão em relação a algo desconhecido ou estranho que pode acarretar desconforto psicológico e/ou físico caso ocorra de forma exacerbada e que, segundo a Organização Mundial da Saúde, afeta significativamente a população brasileira. É importante lidar com este transtorno com o objetivo de diminuir os danos físicos e psicológicos que ele causa nos indivíduos; para isso, as estratégias de tratamento mais utilizadas são a intervenção medicamentosa e tratamentos não farmacológicos como a terapia, atividade física e a meditação. OBJETIVO: Estudar os efeitos da meditação no tratamento da ansiedade. METODOLOGIA: Trata-se de uma revisão sistemática de estudos intervencionais. As bases MEDLINE (Pubmed) e SCIELO foram utilizadas para a coleta de artigos em inglês e português, publicados entre os anos de 2010 e 2020. RESULTADOS: Oito ensaios clínicos randomizados foram selecionados. Os estudos sugerem uma associação positiva entre a prática da meditação e a melhora dos sintomas físicos e psicológicos dos pacientes portadores de transtorno de ansiedade. CONCLUSÃO: De modo geral, a literatura científica evidencia os efeitos benéficos da meditação no tratamento da ansiedade, embora se façam necessárias mais pesquisas sobre o tema.
\end{abstract}

PALAVRAS-CHAVE: Meditação. Ansiedade. Estresse. Qualidade de vida.
ABSTRACT | INTRODUCTION: Anxiety is a subjective state of fear, apprehension, or tension in relation to something unknown or strange that can cause psychological and / or physical discomfort if it occurs in an exacerbated way and, according to the World Health Organization, that represents significant bad effects to the Brazilian population. It is important to deal with this disorder to reduce the physical and psychological damage it causes to individuals; for this, the most used treatment strategies are drug intervention and non-pharmacological treatments such as therapy, physical activity, and meditation. OBJECTIVE: To study the effects of meditation in the treatment of anxiety. METHODOLOGY: This is a systematic review of interventional studies. The MEDLINE (Pubmed) and SCIELO databases were used to collect articles in English and Portuguese, published between 2010 and 2020. RESULTS: Eight randomized clinical trials were selected. Studies suggest a positive association between meditation practice and improved physical and psychological symptoms of patients with anxiety disorder. CONCLUSION: In general, the scientific literature highlights the beneficial effects of meditation in treating anxiety, although more research on the topic is needed.

KEYWORD: Meditation. Anxiety. Stress. Quality of Life. 


\section{Introdução}

A ansiedade é definida por alguns autores como um estado subjetivo de medo, apreensão ou tensão em relação a algo desconhecido ou estranho, que, ao ocorrer de forma exacerbada, causa desconforto psicológico e/ou físico (Gonçalves et al., 2017).

O Manual Diagnóstico e Estatístico de Transtornos Mentais em sua quinta edição (DSM-5) define o medo como a resposta emocional a uma ameaça iminente, real ou percebida; e a ansiedade como a antecipação de uma ameaça futura (DSM-5, 2014). Dessa forma, a ansiedade caracteriza-se como um sentimento vago e desagradável de medo e apreensão, associado a tensão ou desconforto derivado da antecipação de um perigo, de algo desconhecido ou estranho (Mayer, 2017). Manifesta-se fisicamente através de taquicardia, sudorese, tensão muscular, aumento das secreções (urinárias e fecais), aumento da motilidade intestinal e cefaleia (Naue \& Welter, 2017).

Todo ser humano possui ansiedade em níveis naturais (Mayer, 2017); entretanto, ela pode ocorrer de forma exacerbada e ser considerada patológica quando suas manifestações são exageradas e desproporcionais em relação ao estímulo. Nestes casos, ser ansioso passa a interferir na qualidade de vida e no desempenho diário das atividades do indivíduo, em alguns casos, limitando sua atuação (DSM-5, 2014).

Quando considerada como um sintoma persistente, excessivo e aparentemente incontrolável, passa a caracterizar o Transtorno de Ansiedade que segundo o DSM-5 engloba uma série de patologias, entre elas: Transtorno de Ansiedade Generalizada (TAG), Síndrome do Pânico, Transtorno de ansiedade social ou Fobia social, entre outros (DSM-5, 2014). Nesses casos, as manifestações físicas passam a ser mais intensas e associadas a irritabilidade, insônia, dores de cabeça, diarreia, sensação de estar perdendo o controle e comportamentos de evitação (Naue \& Welter, 2017).

Segundo a Organização Mundial da Saúde (OMS), a prevalência mundial do transtorno de ansiedade (TA) é de 3,6\% e está presente em 9,3\% da população brasileira, local que concentra a maior prevalência dos casos de ansiedade entre os países que foram avaliados (World Health Organization; 2017).
Diante deste cenário nacional, as Práticas Integrativas e Complementares em Saúde (PICS) surgiram como uma ampliação das ofertas de cuidado, ao proporcionar uma visão mais abrangente do processo de adoecimento, associada a uma promoção global do cuidado humano, especialmente do autocuidado. Essa visão proporciona maior integralidade e resolutividade na atenção à saúde, assim como aumenta as possibilidades terapêuticas para os usuários do Sistema Único de Saúde (SUS) (Ministério da Saúde, 2018).

Denominadas também como Medicina Tradicional e Complementar/Alternativa pela OMS, as PICS passaram a fazer parte do SUS em 2006 a partir da Política Nacional de Práticas Integrativas e Complementares (PNPIC) na Atenção Básica e demais níveis de saúde. Quando implantada, reunia 5 modalidades de intervenção e ao longo dos anos passou a abranger 29 práticas integrativas, entre elas a Meditação (Ministério da Saúde, 2018).

A meditação pode ser definida como uma prática de integração mente-corpo baseada na vivência do momento presente, com consciência plena e não julgadora a cada instante (Demarzo, 2011). Dessa forma, promove ao indivíduo uma atitude em que a mente fique mais concentrada, atenta e, consequentemente, mais calma (Pereira, 2018). As técnicas meditativas surgiram nas práticas espiritualistas de diversas culturas asiáticas. As primeiras referências conhecidas foram encontradas na Índia relacionadas aos textos do Hinduísmo. Há indícios também de que na China formas tão antigas de meditação se desenvolveram no contexto de tradições nativas, anteriores à codificação do Taoísmo (Menezes et al., 2011; Murphy et al., 1997).

O ato de meditar pode agir para além da dimensão cognitiva e provocar alterações fisiológicas expressivas que interferem diretamente no fluxo mental, desempenhando efeitos positivos nos processos saúde-doença, na qualidade de vida e no bem-estar (Pereira, 2018). As alterações fisiológicas relacionadas a essas práticas são redução da frequência cardíaca, redução da temperatura corporal, aumento do fluxo sanguíneo cerebral, entre outras (Demarzo, 2011; Pereira, 2018).

Enquanto no Oriente meditar é sinônimo de busca espiritual, no Ocidente, em especial nas pesquisas científicas, a palavra meditação tem sido utilizada para descrever práticas autorregulatórias do corpo 
e da mente. A investigação científica da meditação parte da premissa que, embora existam diversas técnicas, todas têm uma característica fundamental em comum: o controle da atenção (Cahn \& Polich, 2006; Goleman, 1988).

A meditação pode ser classificada em três tipos principais na visão ocidental (Cahn \& Polich, 2006; Davidson \& Goleman, 1977): o tipo Mindfulness, em que há uma percepção dos estímulos, como pensamentos, sentimentos e/ou sensações, e observação livre que não os julga nem analisa; as técnicas meditativas concentrativas, que restringem a atenção a um único objeto, interno ou externo, ignorando estímulos do ambiente (Cahn \& Polich, 2006); e a técnica contemplativa, que integra os outros dois tipos, visto que requer tanto a habilidade de focalizar como de se abrir (Shapiro, 1981; Shapiro, Schwartz et al., 2005).

No sentido de atenuar o estresse e a ansiedade, surgiu dentro da modalidade Mindfulness, o MindfulnessBased Stress Reduction (MBSR), um programa organizado em grupos com duração de 8 semanas. Ao longo desse período são realizadas atividades como: prática de meditação guiada, alongamento e prática de yoga voltadas para aumentar a percepção durante a vida diária (Mouzinho et al., 2018).

O treinamento em meditação, uma vez que proporciona ao praticante uma maior capacidade de atenção ao executar uma tarefa, o condiciona a lidar com os fatores ansietogênicos de modo que consiga romper o espiral da reação de enfrentamento ou fuga. Dessa forma, após ter superado um desafio ele apresenta menor predisposição a encarar o próximo compromisso como uma ameaça, como acontece com uma pessoa ansiosa (Goleman, 1999).

É sabido que a prática meditativa regular contribui para a prevenção e tratamento de diversas doenças e condições clínicas e relaciona-se diretamente com o aumento da qualidade de vida e do estado de saúde dos indivíduos, assim como a redução dos níveis prejudiciais de estresse (Demarzo, 2011; Pereira, 2018).

Por conseguinte, visto que ainda não existe consenso sobre o que seria mais efetivo no cuidado da ansiedade: o uso da meditação como terapêutica exclusiva ou associada a tratamentos convencionais; o presente trabalho tem como objetivo analisar a literatura com o intuito de identificar os benefícios da prática regular de meditação nos pacientes que apresentam sintomatologia compatível com os transtornos de ansiedade e como esta prática acarreta em uma melhora na qualidade de vida destes indivíduos.

\section{Justificativa}

A humanidade vive em um contexto no qual existe uma urgência em atender demandas de tarefas, de forma simultânea, relacionadas principalmente ao trabalho, estudo e família (Araújo, 2018). Essas condições, por sua vez, propiciam a um aumento no adoecimento físico e mental da população, levando, principalmente ao desenvolvimento dos transtornos de atenção e de humor, como ansiedade e depressão (Araújo, 2018).

No Brasil, conforme dados da Organização Mundial da Saúde (OMS), a prevalência de quadros de ansiedade é de 18.657 .943 casos, o que equivale a 9,3\% da população, sendo a maior do mundo (Silva, 2018).

Existem diferentes formas de "tratar" quadros de ansiedade, sendo as mais comuns a intervenção medicamentosa e a psicoterápica (Silva, 2018). A busca por tratamento e prevenção dos quadros crônicos de transtornos agravados por estresse tem feito uso de práticas integrativas e complementares, como a meditação, para a intervenção profilática e terapêutica segura, eficaz e efetiva na área da saúde associada ao uso de fármacos (Demarzo, 2011; Araújo, 2018).

Apesar dos inúmeros benefícios e da simplicidade das técnicas meditativas, é necessário ter consciência de que estas não devem ser conduzidas sem o acompanhamento de médicos e outros profissionais da saúde e/ou especialistas na área, visto que as terapias complementares não podem substituir em todos os casos os tratamentos convencionais nem são isentas de riscos, principalmente para os praticantes principiantes e para os portadores de condições clínicas específicas (Demarzo, 2011).

Desta forma, diante da relevância deste tema no cenário nacional, o presente estudo faz-se necessário para demonstrar a importância do uso da meditação adequada, objetivando a diminuição dos sintomas dos transtornos de ansiedade e melhora na qualidade de vida dos pacientes. 


\section{Metodologia}

\section{Desenho de Estudo}

Trata-se de uma revisão sistemática da literatura fundamentada nas recomendações do PRISMA STATEMENT (Liberati et al., 2009).

\section{Critérios de Inclusão}

Foram elegíveis ensaios clínicos envolvendo pacientes portadores de Transtorno de Ansiedade, na faixa etária dos 19 aos 44 anos, submetidos ao tratamento com meditação, e/ou exercício físico. Os estudos incluídos foram realizados entre os anos de 2010 e 2020, escritos em língua inglesa e portuguesa e presentes nas seguintes bases de dados: MEDLINE (Pubmed) e SCIELO.

\section{Critérios de Exclusão}

Foram excluídos trabalhos de revisão sistemática com temas correlatos a este, bem como artigos que não apresentaram compatibilidade temática/titular com o tema proposto.

\section{Fontes de Informação}

As fontes de informação utilizadas na busca foram as seguintes plataformas: MEDLINE (Pubmed) e SCIELO, a última busca foi realizada no dia 19 de junho de 2020.

\section{Estratégia de Busca}

Foi realizada uma busca avançada em cada uma das fontes de informação supracitadas. As palavras-chave foram identificadas a partir do MeSH ${ }^{\circledR}$ Headings e utilizados da seguinte forma com os operadores boleanos: ("Meditation") AND ("Anxiety") AND ("Aerobic exercise"). Em seguida foram utilizados os filtros para o tipo de estudo, idioma, data da publicação e idade.

\section{Seleção dos Estudos}

A seleção dos estudos foi feita por meio de três triagens: triagem por títulos, por resumos e pela leitura completa do artigo. Em seguida, foram selecionados de forma independente, os estudos que preencheram os critérios de inclusão e que possuíam compatibilidade temática com o escopo desta revisão.
Visando garantir a qualidade metodológica do estudo, todos os artigos selecionados foram submetidos à Escala PEDro (https://pedro.org.au/wp-content/ uploads/PEDro_scale_portuguese(brasil).pdf) sendo incluídos aqueles com score igual ou superior a 5 . Após confrontamento de informações dos revisores e resolução de discordâncias por consenso, os artigos foram inseridos nesta revisão sistemática.

Atualmente, a escala mais usada na área da reabilitação é a escala PEDro. Ela foi desenvolvida pela Physiotherapy Evidence Database para ser empregada em estudos experimentais e tem uma pontuação total de até 10 pontos, incluindo critérios de avaliação de validade interna e apresentação da análise estatística empregada. Para cada critério definido na escala, um ponto (1) é atribuído à presença de indicadores da qualidade da evidência apresentada, e zero ponto (0) é atribuído à ausência desses indicadores. A escala PEDro é composta pelos seguintes critérios: 1) especificação dos critérios de inclusão (item não pontuado); 2) alocação aleatória; 3) sigilo na alocação; 4) similaridade dos grupos na fase inicial ou basal; 5) mascaramento dos sujeitos; 6) mascaramento do terapeuta; 7) mascaramento do avaliador; 8) medida de pelo menos um desfecho primário em $85 \%$ dos sujeitos alocados; 9) análise da intenção de tratar; 10) comparação entre grupos de pelo menos um desfecho primário e 11) relato de medidas de variabilidade e estimativa dos parâmetros de pelo menos uma variável primária.

\section{Processo de Coleta de Dados}

A extração dos dados ocorreu de forma independente, através da realização de uma tabela-síntese composta pelos itens descritos na estratégia PICO, sendo a População: pacientes portadores de ansiedade, Intervenção: meditação, Comparação: exercício físico, Desfecho: redução da ansiedade e Tipo de estudo: ensaios clínicos.

\section{Lista de Dados}

Os dados dos artigos selecionados atendendo aos critérios de inclusão, foram coletados através de uma tabela composta pelos seguintes itens: autor, data de publicação, local de estudo, número de pacientes, idade e tempo de meditação, principais desfechos, resultados encontrados e limitações dos estudos. 


\section{Aspectos éticos}

Por se tratar de uma revisão sistemática, o presente estudo não será submetido à avaliação do Comitê de Ética em Pesquisa de acordo com a Resolução 466/12 do Conselho Nacional de Saúde (CNS), isenta-se de termo esclarecido de consentimento e submissão ao CEP. Contudo, todos os preceitos éticos estabelecidos serão respeitados no que se refere a zelar pela legitimidade das informações e direitos autorais, quando necessárias, tornando os resultados desta pesquisa públicos.

\section{Resultados}

Foram identificados 156 artigos através da pesquisa nas bases de dados PubMed e SciELO. Dentre os artigos encontrados, 49 foram selecionados através da leitura dos títulos e resumo, sendo excluídos 35 artigos por não atenderem os critérios de inclusão. Na terceira triagem, mais 6 foram excluidos por conta dos textos completos estarem indisponíveis, impossibilitndo a leitura completa. Por fim, um total de 8 artigos foram incluídos na revisão sistemática. O algoritmo da seleção está representado na Figura 1.

Figura 1. Representação esquemática dos métodos de identificação, triagem, elegibilidade e inclusão de artigos na revisão, adaptada de acordo com o PRISMA Flow Diagram

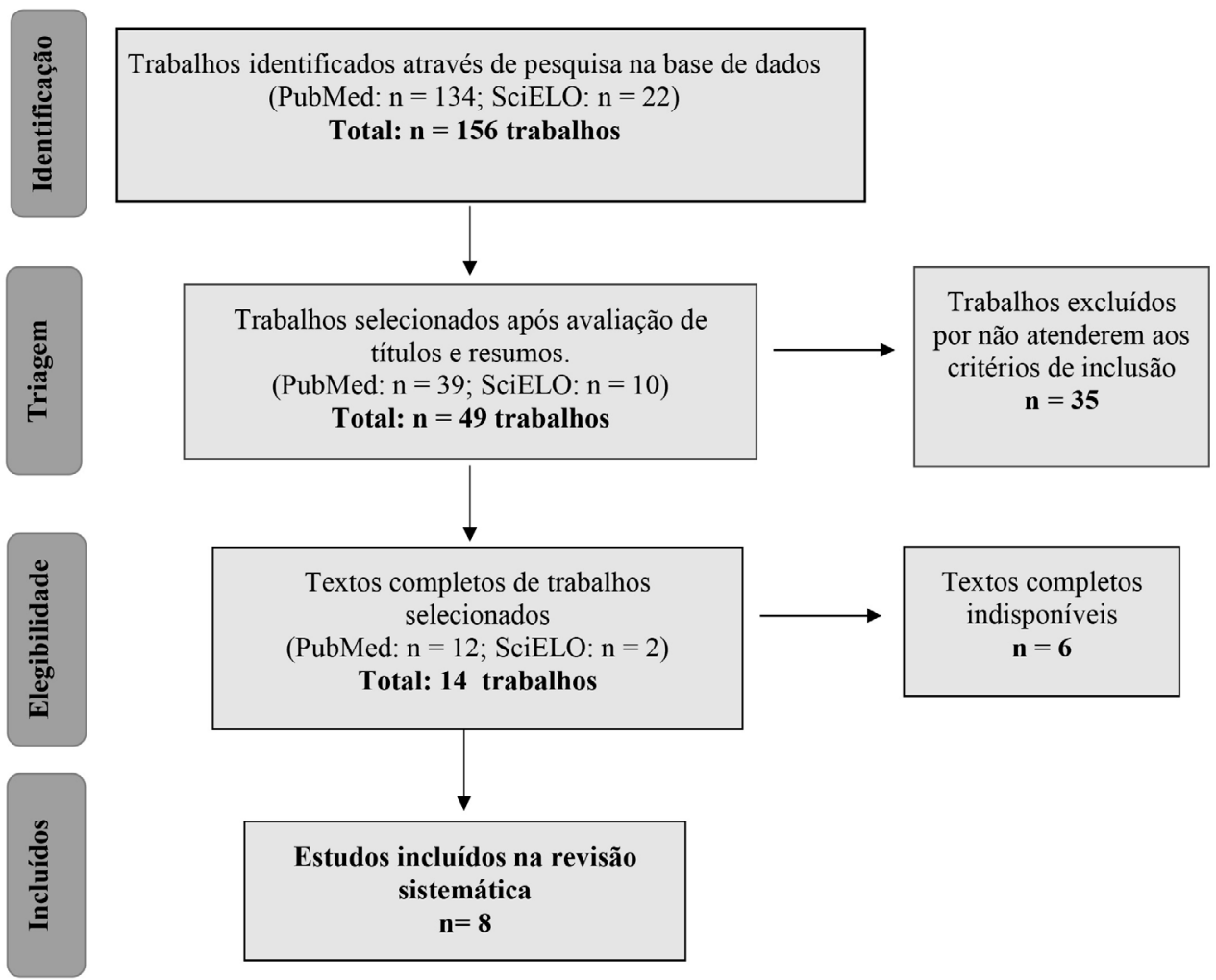

Os resultados serão apresentados nas três tabelas abaixo, sendo que $\mathrm{n}$ representa o número de pacientes estudados, a idade estará em anos, todos os resultados foram retirados de ensaio clínico randomizado (ECR), sendo que o programa de meditação Mindfulness Based Stress Reduction. (MBSR) esteve presente na maioria dos artigos. 
Quadro 1. Efetividade da meditação no tratamento da ansiedade

\begin{tabular}{|l|l|l|l|l|l|l|}
\hline \multicolumn{1}{|c|}{ Autor } & n & Idade & Estudo & $\begin{array}{l}\text { Tempo e } \\
\text { programa de } \\
\text { meditação }\end{array}$ & \multicolumn{1}{c|}{ Desfecho } & \multicolumn{1}{c|}{ Conclusão } \\
\hline $\begin{array}{l}\text { Menezes, C. et } \\
\text { al. (2016) }\end{array}$ & 31 & $20-40$ & ECR & $\begin{array}{l}5 \text { dias consecutivos } \\
\text { de meditação } \\
\text { concentrativa } \\
\text { (média de } 20 \\
\text { min/dia) }\end{array}$ & $\begin{array}{l}\text { A meditação focada foi capaz } \\
\text { de reduzir a ansiedade, regular } \\
\text { a atenção, melhorar o estado } \\
\text { basal, promover equilíbrio e } \\
\text { relaxamento. }\end{array}$ & $\begin{array}{l}\text { O treinamento de } 5 \text { dias em } \\
\text { meditação focada promoveu } \\
\text { habilidades de regulação emocional, } \\
\text { modulando a interação entre } \\
\text { interferência emocional e ansiedade. }\end{array}$ \\
\hline $\begin{array}{l}\text { Menezes, C., } \\
\text { Bizarro, L. } \\
\text { (2015) }\end{array}$ & 33 & $20-40$ & ECR & $\begin{array}{l}5 \text { dias consecutivos } \\
\text { de meditação } \\
\text { concentrativa } \\
\text { (média de 20 } \\
\text { min/dia) }\end{array}$ & $\begin{array}{l}\text { O treinamento de meditação } \\
\text { produziu redução da } \\
\text { ansiedade, dos afetos } \\
\text { negativos e aumentou } \\
\text { potencial de concentração. }\end{array}$ & $\begin{array}{l}\text { Um breve treinamento em } \\
\text { meditação focada melhora o humor, } \\
\text { confiança e bem-estar e um maior } \\
\text { tempo de prática se traduz em } \\
\text { maior benefício frente a fatores } \\
\text { ansietogênicos. }\end{array}$ \\
\hline $\begin{array}{l}\text { Hoge, E. et al. } \\
\text { (2014). }\end{array}$ & 89 & $25-55$ & ECR & $\begin{array}{l}8 \text { semanas de } \\
\text { MBSR } \\
\text { (média de 45 } \\
\text { min/dia) }\end{array}$ & $\begin{array}{l}\text { A meditação Mindfulness foi } \\
66 \% \text { melhor na redução da } \\
\text { ansiedade e angústia do que o } \\
\text { programa de ensinamento de } \\
\text { controle ativo contra o } \\
\text { estresse. }\end{array}$ & $\begin{array}{l}\text { A redução do estresse com base na } \\
\text { Mindfulness demonstrou ser uma } \\
\text { ferramenta eficaz para reduzir a } \\
\text { ansiedade em pacientes com } \\
\text { Transtorno da Ansiedade } \\
\text { Generalizada (TAG). Além disso, os } \\
\text { pacientes que aprenderam } \\
\text { meditação Mindfulness tiveram } \\
\text { menos reatividade ao estresse e } \\
\text { foram mais resistentes a uma tarefa } \\
\text { de estresse laboratorial. }\end{array}$ \\
\hline
\end{tabular}

Quadro 2. Efetividade da meditação na qualidade de vida

\begin{tabular}{|l|l|l|l|l|l|l|}
\hline \multicolumn{1}{|c|}{ Autor } & n & Idade & Estudo & $\begin{array}{l}\text { Tempo e programa } \\
\text { de meditação }\end{array}$ & \multicolumn{1}{c|}{ Desfecho } & \multicolumn{1}{c|}{ Conclusão } \\
\hline $\begin{array}{l}\text { Hoge, E. et al. } \\
\text { (2018) }\end{array}$ & 70 & $26-54$ & ECR & 8 semanas de MBSR & $\begin{array}{l}\text { Pacientes submetidos a MBSR } \\
\text { tiveram uma redução nas } \\
\text { taxas do Hormônio } \\
\text { Adrenocorticotrófico (ACTH) } \\
\text { bem como das citocinas } \\
\text { inflamatórias, quando } \\
\text { comparado aos não } \\
\text { meditadores. }\end{array}$ & $\begin{array}{l}\text { O treinamento em Mindfulness é } \\
\text { útil para diminuir a reatividade } \\
\text { biológica ao estresse e melhorar a } \\
\text { resistência a estressores em } \\
\text { pacientes com Transtorno da } \\
\text { Ansiedade Generalizada (TAG). }\end{array}$ \\
\hline $\begin{array}{l}\text { Boettcher, J. } \\
\text { et al. (2014) }\end{array}$ & 91 & $18-44$ & ECR & $\begin{array}{l}8 \text { semanas } \\
\text { de MBSR } \\
\text { (média de 17 } \\
\text { min/dia) }\end{array}$ & $\begin{array}{l}\text { A meditação Mindfulness } \\
\text { promoveu resultados positivos } \\
\text { no tratamento de pessoas com } \\
\text { transtornos de ansiedade } \\
\text { heterogêneos quando tratadas } \\
\text { de forma remota, através da } \\
\text { internet. }\end{array}$ & $\begin{array}{l}\text { Os efeitos positivos da meditação } \\
\text { remotos, via internet, foram } \\
\text { semelhantes àquelas } \\
\text { apresentadas pelos pacientes que } \\
\text { realizam a terapia de forma } \\
\text { presencial. }\end{array}$ \\
\hline $\begin{array}{l}\text { Mcmanus, F. } \\
\text { et al. (2012) }\end{array}$ & 74 & $18-65$ & ECR & $\begin{array}{l}\text { 8 semanas de MBSR } \\
\text { adaptado para } \\
\text { Transtorno de } \\
\text { Ansiedade de Doença }\end{array}$ & $\begin{array}{l}\text { Houve benefício no uso de } \\
\text { meditação Mindfulness para o } \\
\text { tratamento de pacientes com } \\
\text { hipocondria imediatamente } \\
\text { após a intervenção, e, 1 ano } \\
\text { após. }\end{array}$ & $\begin{array}{l}\text { A meditação Mindfulness pode ser } \\
\text { uma adição útil aos serviços usuais } \\
\text { para pacientes com hipocondria. }\end{array}$ \\
\hline
\end{tabular}


Quadro 3. Efetividade da meditação de forma isolada e em associação ao exercício aeróbico

\begin{tabular}{|c|c|c|c|c|c|c|}
\hline Autor & $\mathbf{n}$ & Idade & Estudo & $\begin{array}{l}\text { Tempo e programa } \\
\text { de meditação }\end{array}$ & Desfecho & Conclusão \\
\hline $\begin{array}{l}\text { Jazaieri, H. } \\
\text { et al. (2012) }\end{array}$ & 133 & $24-41$ & ECR & 8 semanas de MBSR & $\begin{array}{l}\text { A redução do estresse baseado } \\
\text { na meditação Mindfulness e a } \\
\text { realização de exercícios } \\
\text { aeróbicos foram associados a } \\
\text { redução do Transtorno de } \\
\text { Ansiedade Social e Depressão, e } \\
\text { ao aumento no bem-estar } \\
\text { subjetivo. }\end{array}$ & $\begin{array}{l}\text { Mindfulness e Exercício Aeróbico } \\
\text { associados, demonstrou melhoria } \\
\text { nas medidas de sintomas clínicos e } \\
\text { bem-estar dos pacientes com } \\
\text { Transtorno de Ansiedade Social } \\
\text { (TAS), quando comparado com o } \\
\text { grupo de pacientes com TAS não } \\
\text { tratados. }\end{array}$ \\
\hline $\begin{array}{l}\text { Goldin, P. } \\
\text { et al. (2012) }\end{array}$ & 49 & $24-41$ & ECR & 8 semanas de MBSR & $\begin{array}{l}\text { Alteração na regulação da } \\
\text { atenção durante a meditação } \\
\text { Mindfulness beneficiou pacientes } \\
\text { com transtorno de ansiedade, } \\
\text { pois aumentou a capacidade de } \\
\text { exercer controles cognitivos } \\
\text { sobre ruminação negativa, maior } \\
\text { capacidade de desvio da atenção } \\
\text { ao estado de ansiedade e } \\
\text { redução da reatividade } \\
\text { emocional. }\end{array}$ & $\begin{array}{l}\text { Houve maior redução na emoção } \\
\text { negativa e na gravidade dos } \\
\text { sintomas em paciente com } \\
\text { transtorno de ansiedade } \\
\text { generalizado, que realizaram a } \\
\text { meditação, além de implementar } \\
\text { aumento nas regiões corticais } \\
\text { parietais relacionadas à atenção e } \\
\text { a regulação de crenças pessoais } \\
\text { negativas quando comparada com } \\
\text { exercício aeróbico. }\end{array}$ \\
\hline
\end{tabular}

\section{Discussão}

Entre os oito ensaios clínicos randomizados incluídos na presente revisão sistemática, quatro destes foram realizados nos Estados Unidos da América (EUA) (Hoge et al., 2014; Hoge et al., 2018; Jazaieri et al., 2012; Goldin et al., 2012), dois no Brasil (Menezes, Pereira, Mocaiber \& Bizarro, 2016; Menezes \& Bizarro, 2015), um na Alemanha (Boettcher et al., 2014) e um na Inglaterra (McManus et al., 2012). A preponderância de estudos em países desenvolvidos decorre de dois fatores: maiores investimentos em pesquisas nestas regiões, por disponibilizarem mais recursos; e, elevada prevalência de pacientes com transtornos de ansiedade heterogêneos na população, associada a uma lacuna de 30-40\% de não adesão ou resolutividade da patologia por meio dos tratamentos convencionais, farmacológicos ou não (Hoge et al., 2014; Hoge et al., 2018; Boettcher et al., (2014); Jazaieri et al., 2012; Goldin et al., 2012).

No que tange à população estudada, os artigos dispuseram de um total de 570 participantes; o maior foi conduzido por Jazaieri et al. (2012), nos EUA, com 133 pacientes e o menor com 31 pacientes realizado no Brasil por Carolina BM et al.

Quanto ao tipo de meditação utilizado, houve uma predominância pela Mindfulness (Hoge et al., 2014; Hoge et al., 2018; Boettcher et al., 2014; McManus et al., 2012; Jazaieri et al., 2012; Goldin et al., 2012), que consiste em auxiliar o indivíduo a desenvolver uma percepção aberta e não reativa aos eventos cotidianos, associado à capacidade de processar as experiências de vida sem tentar controlar, suprimir ou evitar sensações e situações (Boettcher et al., 2014). Dentro desta modalidade de meditação desenvolveu- -se um programa focado na redução do estresse, Mindfulness-Based Stress Reduction (MBSR), organizado em 8 semanas, que envolve o cultivo de um foco de atenção não julgador, flexível e do momento presente (Jazaieri et al., 2012; Goldin et al., 2012). No modelo padrão, o MBSR consiste em 8 aulas semanais em grupo, com duração de 2,5 horas, um retiro de meditação de um dia e 40 minutos de prática diária em casa (Hoge et al., 2018; Jazaieri et al., 2012; Goldin et al., 2012). 
Três dos artigos revisados utilizaram este método padrão de Mindfulness (Hoge et al., 2018; Jazaieri et al., 2012; Goldin et al., 2012), comparado a outras terapêuticas alternativas para a ansiedade, entre elas, a realização de exercício aeróbico (Jazaieri et al., 2012; Goldin et al., 2012), e a realização de treinamento para controle da atenção (Hoge et al., 2018). Em outros três trabalhos, o MBSR passou por adaptações para avaliar seus benefícios no tratamento de hipocondria (ansiedade de saúde) (McManus et al., 2012), assim como sua aplicabilidade de forma remota, via internet (Boettcher et al., 2014), e o uso da meditação na reatividade ao estresse com base na atenção plena (Hoge et al., 2014) comparado com terapias "convencionais" para a ansiedade, como programas de educação em gerenciamento de estresse e terapias cognitivo-comportamentais (Hoge et al., (2014); Boettcher et al., (2014); McManus et al., 2012).

Todos os artigos que utilizaram a modalidade Mindfulness conseguiram associar o método MBSR à diminuição da tendência habitual de reagir emocionalmente e ruminar sobre pensamentos transitórios e sensações físicas, estresse e auto visão distorcida (Jazaieri et al., 2012). Observou-se também que há uma redução nos sintomas de depressão, ansiedade e melhora do humor e da qualidade de vida dos pacientes após o tratamento com Mindfulness (Boettcher et al., 2014; Goldin et al., 2012). É digno de nota que o MBSR pode aumentar a resistência biológica ao estresse, por influenciar na secreção dos hormônios relacionados a esta sensação, como o ACTH, assim como na presença de marcadores inflamatórios, como o Fator de Necrose Tumoral alfa (TNF-alfa) e a Interleucina 6 (IL-6) que podem ser associados ao risco de doenças cardiovasculares e síndrome metabólica a longo prazo (Hoge et al., (2018)).

A outra modalidade de meditação avaliada nos trabalhos foi a Concentrativa, por um período de treinamento curto de 5 dias, com tempo médio de 20 minutos de meditação diária (Menezes et al., 2016; Menezes \& Bizarro, 2015). A sua aplicabilidade foi analisada em pacientes sem diagnóstico de transtorno de ansiedade, mas que apresentavam sintomas de estresse, irritabilidade e hiper-reatividade (Menezes et al., 2016; Menezes \& Bizarro, 2015). Os resultados indicaram uma diminuição da reatividade a estímulos negativos, melhora da capacidade de desviar a atenção do processo de associação e reação automática aos pensamentos, emoções e sentimentos, assim como redução do afeto negativo e da ansiedade (Menezes et al., 2016; Menezes \& Bizarro, 2015).

A associação da meditação ao tratamento medicamentoso padrão para os transtornos de ansiedade não foi analisada diretamente por nenhum artigo. Seus benefícios foram equivalentes e em algumas vezes superiores às terapêuticas alternativas utilizadas, como o exercício aeróbico e a terapia cognitiva-comportamental (Hoge et al., 2014; Hoge et al., 2018; Boettcher et al., 2014; McManus et al., 2012; Goldin et al., 2012) e significativamente maior quando comparados com pacientes não tratados (Jazaieri et al., 2012).

A meditação foi altamente aceita e teve efeitos positivos nos pacientes, tanto físicos quanto psicológicos (Menezes \& Bizarro, 2015; Hoge et al., (2018)), mostrando-se uma terapia eficaz e bem tolerada, sem grandes eventos adversos, mesmo com um período relativamente curto de prática (Menezes et al., 2016; Menezes \& Bizarro, 2015). Estes efeitos precoces funcionam como motivadores, e auxiliam na manutenção e regularidade da prática e, consequentemente na obtenção dos benefícios psicológicos (Menezes \& Bizarro, 2015).

Quanto ao tipo de meditação e o tempo necessário para as sessões, a quantidade ideal ainda não se encontra bem documentada na literatura, visto que os estudos mostraram benefícios desde períodos curtos de 5 dias (Menezes et al., 2016; Menezes \& Bizarro, 2015) de treinamento até períodos mais longos de 8 semanas (Hoge et al., 2014; Hoge et al., 2018; Boettcher et al., 2014; McManus et al., 2012; Jazaieri et al., 2012; Goldin et al., 2012) e os mantiveram mesmo após um tempo de 3 e 12 meses (Boettcher et al., 2014; Jazaieri et al., 2012). Por conta disso há a necessidade de mais estudos que comparem o tipo de meditação escolhida, com as condições clínicas específicas dos pacientes e o tempo necessário para a apresentação dos benefícios da prática (Menezes \& Bizarro, 2015; Boettcher et al., 2014; Jazaieri et al., 2012; Goldin et al., 2012).

A meditação por si só é capaz de promover redução no estresse, na ansiedade social, nos sintomas de depressão e aumentar o bem-estar subjetivo e a qualidade de vida dos indivíduos (Jazaieri et al., 2012). O estresse psicológico, por sua vez, pode contribuir para o início, exacerbação ou recaída dos transtornos de ansiedade. Por conta disso, os pacientes são muito beneficiados com a possibilidade de um tratamento 
alternativo, como a meditação, por se tratar de uma abordagem de baixo custo, acessível e de baixo estigma (Hoge et al., 2018), para aqueles que não desejam participar de tratamentos tradicionais ou não respondem ao uso das terapêuticas convencionais (Jazaieri et al., 2012). Uma terapia bem-sucedida pode resultar em melhora da saúde psicológica e física, e aumentar ainda mais a relevância do tratamento de transtorno de ansiedade para a saúde pública (Hoge et al., 2018).

\section{Limitações e perspectivas}

No que diz respeito à metodologia utilizada, todos os ensaios clínicos analisados apresentaram limitações características desse desenho de estudo, podendo a acurácia dos resultados encontrados ser comprometida. Dentre as limitações mais comuns estão: pequena amostragem (Menezes et al., 2016; Menezes \& Bizarro, 2015; Hoge et al., 2014; Hoge et al., 2018), falta de grupo controle ativo (Menezes et al., 2016; Menezes \& Bizarro, 2015; Hoge et al., 2014; McManus et al., 2012; Jazaieri et al., 2012), amostra composta por grupos pouco variados e não generalizáveis, e falta de acompanhamento quando a intervenção era finalizada (Menezes et al., 2016; Menezes \& Bizarro, 2015; Boettcher et al., 2014).

Destacamos ainda a necessidade de serem realizados estudos cujo grupo que realize tratamento apenas com meditação possa ser comparado com um grupo que utilize apenas terapia convencional, além da comparação da associação entre ambos. Salientamos também que não foram encontrados Estudos Clínicos Randomizados envolvendo tal tema nas plataformas utilizadas. Destacamos ainda a necessidade de realizar estudos envolvendo maior número de pacientes, de modo que seja possível generalizar os resultados encontrados.

\section{Conclusão}

Os estudos analisados nesta revisão apontaram para uma redução dos sintomas nos pacientes que praticaram a meditação, associada ou não a outras terapias convencionais utilizadas no tratamento dos transtornos de ansiedade heterogêneos. Desta forma, a partir desta revisão, podemos concluir que a literatura científica sustenta os benefícios da realização da meditação no tratamento da ansiedade para melhora da qualidade de vida destes pacientes, sendo que este dado foi observado em $100 \%$ dos artigos selecionados para esta revisão sistemática.

\section{Contribuições dos autores}

Almeida Júnior, I. A. J. participou da concepção, delineamento e redação do artigo. Guimarães, B. e Peixoto, J. L. participaram da concepção, delineamento, busca e análise dos dados da pesquisa, interpretação dos resultados, redação do artigo científico. Pires MD participou da concepção, delineamento, redação do artigo e interpretação dos dados da pesquisa.

\section{Conflitos de interesses}

Nenhum conflito financeiro, legal ou político envolvendo terceiros (governo, empresas e fundações privadas, etc.) foi declarado para nenhum aspecto do trabalho submetido (incluindo, mas não se limitando a subvenções e financiamentos, participação em conselho consultivo, desenho de estudo, preparação de manuscrito, análise estatística, etc.).

\section{Referências}

American Psychiatric Association. (2014). Manual diagnóstico e estatístico de transtornos mentais: DSM-5 (5 5 d.). Artmed.

Araújo, G. L. L. (2018). Efeitos psicofisiológicos de uma breve intervenção baseada em mindfulness em adultos jovens saudáveis [Tese de doutorado Universidade Federal do Rio Grande do Norte]. Repositório UFRN. https://repositorio. ufrn.br/handle/123456789/26952

Boettcher, J., Astrom, V., Pahlsson, D., Andersson, G., \& Carlbring, P. (2014). Internet-Based Mindfulness Treatment for Anxiety Disorders: A Randomized Controlled Trial [Tratamento de Atenção Baseada na Internet para Transtornos de Ansiedade: Um julgamento controlado aleatorizado]. Behavior Therapy, 45, 241-253. https://doi. org/10.1016/j.beth.2013.11.003

Cahn, B. R., \& Polich, J. (2006). Meditation states and traits: EEG, ERP and neuroimaging studies [Estados e características da meditação: EEG, ERP e estudos de neuroimagem]. Psychological Bulletin, 132(2), 180-211. https://doi. org/10.1037/0033-2909.132.2.180

Chaves, M., \& Corrêa, Y. (2018). Ansiedade e Depressão: dificuldades e caminhos a serem descobertos [Minicurso]. Pet Bio. Unifal. https://docplayer.com.br/145087091-Ansiedade-edepressao-dificuldades-e-caminhos-a-serem-descobertos. html 
Davidson, R. J., \& Goleman, D. J. (1977). The role of attention in meditation and hypnosis: A psychobiological perspective on transformations of consciousness [O papel da atenção na meditação e hipnose: Uma perspectiva psicobiológica sobre as transformações da consciência]. The international Journal of Clinical and Experimental Hypnosis, 25(4), 291 308. https://doi.org/10.1080/00207147708415986

Demarzo, M. M. P. (2011). Meditação aplicada à saúde. Programa de Atualização em Medicina de Família e Comunidade. Artmed, 6, 1-18.

Goldin, P., Ziv, M., Jazaieri, H., Hahn, K., \& Gross, J. (2012). MBSR vs Aerobic Exercise in Social Anxiety: fMRI of Emotion Regulation of Negative Self-Beliefs [MBSR vs Exercício Aeróbico em Ansiedade Social: fMRI de Regulação da Emoção de Autoconfiança Negativa]. Social cognitive and affective neuroscience, 8(1), 65-72. https://doi.org/10.1093/ scan/nss054

Goleman, D. J. (1988). The meditative mind: The varieties of meditative experience [A mente meditativa: As variedades da experiência meditative]. Jeremy R. Tarcher, Inc.

Goleman, D. PhD (1999). A arte da meditação - Um guia para a meditação (4a ed.) Sextante.

Gonçalves, F., Mendes, A., \& Santos, J. V. (2017). Ansiedade e satisfação com a vida. Estudo das relações numa amostra de estudantes portugueses. OMNIA, Revista Interdisciplinar de Ciências e Artes, 7, 23-40. https://www.researchgate. net/profile/Fabio-Goncalves-7/publication/320388551_ Ansiedade_e_satisfacao_com_a_vida_Estudo_das relacoes numa amostra de estudantes portugueses/ links/5afaa561a6fdccacab16e18e/Ansiedade-e-satisfacaocom-a-vida-Estudo-das-relacoes-numa-amostra-deestudantes-portugueses.pdf

Hoge, E. A., Bui, E., Marques, L., Metcalf, C. A., Morris, L. K., Robinaugh, D. J., Worthington, J. J., Pollack, M. H., \& Simon, N. M. (2013). Randomized controlled trial of mindfulness meditation for generalized anxiety disorder: effects on anxiety and stress reactivity [Ensaio randomizado e controlado de meditação de atenção para transtorno de ansiedade generalizada: efeitos sobre a ansiedade e a reatividade ao estresse]. The Journal of clinical psychiatry, 74(8), 786-792. https://doi.org/10.4088/JCP.12m08083

Hoge, E. A., Bui, E., Palitz, S. A., Schwarz, N. R., Owens, M. E., Johnston, J. M., Pollack, M. H., \& Simon, N. M. (2018). The effect of mindfulness meditation training on biological acute stress responses in generalized anxiety disorder [O efeito do treinamento de meditação consciente sobre as respostas biológicas ao estresse agudo em distúrbios de ansiedade generalizada]. Psychiatry research, 262, 328-332. https://doi.org/10.1016/j.psychres.2017.01.006
Jazaieri, H., Goldin, P. R., Werner, K., Ziv, M., \& Gross, J. J. (2012). A randomized trial of MBSR versus aerobic exercise for social anxiety disorder [Um ensaio randomizado de MBSR versus exercício aeróbico para distúrbio de ansiedade social]. Journal of clinical psychology, 68(7), 715-731. https://doi.org/10.1002/jclp.21863

Liberati, A., Altman, D. G., Tetzlaff, J., Mulrow, C., Gøtzsche, P. C., Ioannidis, J. P. A., Clarke, M., Devereaux, P. J., Kleijnen, J., \& Moher, D. (2009). The PRISMA statement for reporting systematic reviews and meta-analyses of studies that evaluate health care interventions: explanation and elaboration [A declaração PRISMA para relatar revisões sistemáticas e meta-análises de estudos que avaliam intervenções de assistência à saúde: explicação e elaboração]. BMJ, 6(7), e1000100. https://doi.org/10.1136/ bmj.b2700

Mayer, F. (2017). A prevalência de sintomas de depressão e ansiedade entre os estudantes de medicina: um estudo multicêntrico no Brasil [Tese de doutorado, Universidade de São Paulo]. Biblioteca Digital USP. https://www.teses. usp.br/teses/disponiveis/5/5169/tde-13112017-154429/ publico/FernandaBrenneisenMayerVersaoCorrigida.pdf

McManus, F., Surawy, C., Muse, K., Vazquez-Montes, M., \& Williams, J. M. (2012). A randomized clinical trial of mindfulness-based cognitive therapy versus unrestricted services for health anxiety (hypochondriasis) [Um ensaio clínico aleatório de terapia cognitiva baseada na atenção versus serviços irrestritos para ansiedade de saúde (hipocondriase)]. Journal of consulting and clinical psychology, 80(5), 817-828. https://doi.org/10.1037/ a0028782

Menezes, C. B., \& Bizarro, L. (2015). Efeitos de um Treino Breve de Meditação no Afeto Negativo, Ansiedade Traço e Atenção Concentrada. Paidéia (Ribeirão Preto), 25, 62. https://doi. org/10.1590/1982-43272562201513

Menezes, C. B., Dell'Aglio, D. D., \& Bizzarro, L. (2011). Meditação, bem-estar e a ciência psicológica: revisão de estudos empíricos. Interação em Psicologia, 15(2), 239-248. http:// dx.doi.org/10.5380/psi.v15i2.20249

Menezes, C. B., \& Dell'Aglio, D. D. (2009). Os efeitos da meditação à luz da investigação científica em Psicologia: revisão de literatura. Psicologia: ciência e profissão, 29(2), 276-289. https://doi.org/10.1590/S1414-98932009000200006

Menezes, C. B., Pereira, M. G., Mocaiber, I., \& Bizarro, L. (2016). Meditação breve e a interação entre interferência emocional e ansiedade. Psicologia: Teoria e Pesquisa, 32(2). https://doi.org/10.1590/0102-3772e322216 
Ministério da Saúde. (2018). Manual de implantação de serviços de práticas integrativas e complementares no SUS. Ministério da Saúde. Secretaria de Atenção à Saúde. Departamento de Atenção Básica. http://189.28.128.100/dab/docs/ portaldab/publicacoes/manual_implantacao_servicos_pics. pdf

Mouzinho, L., Costa, N., Alves, T., Silva, S., \& Lima, L. D. (2018). Contribuições do mindfulness às condições médicas: uma revisão de literatura. Psicologia, saúde \& doenças, 19(2), 182-196. http://dx.doi.org/10.15309/18psd190202

Murphy, M., Donovan, S., \& Taylor, E. (1997). The physical and psychological effects of meditation: A review of contemporary research [Os efeitos físicos e psicológicos da meditação: Uma revisão da pesquisa contemporâne]. Institute of Noetic Sciences.

Naue, C., Welter, M. (2017). Transtorno de ansiedade infantil. Revista Saberes e Sabores Educacionais, (4). https:// docplayer.com.br/122590769-Revista-saberes-e-saboreseducacionais-issn-x-no-4-ano-2017.html

Pereira, A. (2018). A produção científica sobre meditação: Artigos de 2009 a 2018 [Trabalho de conclusão de curso, Universidade Federal de Uberlândia]. Repositório Institucional UFU. https://repositorio.ufu.br/ handle/123456789/23631

Shapiro Jr, D. H. (1981). Psychiatry-Epitomes of Progress: Meditation: Clinical and Health-Related Applications
[Psiquiatria-Epitomas do Progresso: Meditação: Aplicações clínicas e relacionadas com a saúde]. Western Journal of Medicine, 134(2), 141-142. https://www.ncbi.nlm.nih.gov/ pmc/articles/PMC1272535/pdf/westjmed00222-0055b.pdf

Shapiro, S. L., Schwartz, G. E., \& Santerre, C. (2005). Meditation and positive psychology [Meditação e psicologia positiva]. In C. R. Snyder \& S. J. Lopez, Handbook of positive psychology (pp. 632-645). Oxford USA Trade.

Silva, M. (2018). Efeitos de um programa de Mindfulness a estudantes do ensino médio com ansiedade [Trabalho de conclusão de curso, Universidade Federal do Maranhão]. Biblioteca Digital. https://monografias.ufma.br/jspui/ handle/123456789/3086

University library. (2020, Set 8). Evidence Based Medicine [Medicina Baseada em Evidências]. https://researchguides.uic.edu/ ebm

Willhelm, A. R., Andretta, I., \& Ungaretti, M. S. (2015). Importância das técnicas de relaxamento na terapia cognitiva para ansiedade. Contextos Clínicos, 8(1), 79-86. https://doi. org/10.4013/ctc.2015.81.08

World Health Organization. (2017). Depression and Other Common Mental Disorders: Global Health Estimates [Depressão e outros distúrbios mentais comuns: Estimativas de saúde global]. https://apps.who.int/iris/bitstream/ handle/10665/254610/WHO-MSD-MER-2017.2-eng.pdf 